\title{
Erratum: Path-integral description of combined Hamiltonian and non-Hamiltonian dynamics in quantum dissipative systems [Phys. Rev. B 94, 125439 (2016)]
}

\author{
A. M. Barth, A. Vagov, and V. M. Axt \\ (Received 17 August 2017; published 5 September 2017)
}

DOI: 10.1103/PhysRevB.96.119901

In the original paper there is an error in Eq. (23). The corrected equation should read

$$
\rho_{v_{n} \cdots v_{n-n_{c}+1}}^{\mu_{n} \cdots \mu_{n-n_{c}+1}}=\mathcal{M}_{v_{n} \mu_{n}}^{v_{n-1} \mu_{n-1}} \sum_{\substack{v_{n-n_{c}} \\ \mu_{n-n_{c}}}} \exp \left(\sum_{\ell=n-n_{c}}^{n} S_{v_{n} v_{\ell} \mu_{n} \mu_{\ell}}\right) \rho_{v_{n-1} \cdots v_{n-n_{c}}}^{\mu_{n-1} \cdots \mu_{n-n_{c}}} .
$$

This correction does not affect the discussion in the paper. In particular, all the conclusions are unaffected. 